\title{
Dynamic Hall Effect Driven by Circularly Polarized Light in a Graphene Layer
}

\author{
J. Karch, ${ }^{1}$ P. Olbrich, ${ }^{1}$ M. Schmalzbauer, ${ }^{1}$ C. Zoth, ${ }^{1}$ C. Brinsteiner, ${ }^{1}$ M. Fehrenbacher, ${ }^{1}$ U. Wurstbauer, ${ }^{1}$ M. M. Glazov, ${ }^{2}$ \\ S. A. Tarasenko, ${ }^{2}$ E. L. Ivchenko, ${ }^{2}$ D. Weiss, ${ }^{1}$ J. Eroms, ${ }^{1}$ R. Yakimova, ${ }^{3}$ S. Lara-Avila, ${ }^{4}$ S. Kubatkin, ${ }^{4}$ and S. D. Ganichev ${ }^{1}$ \\ ${ }^{1}$ Terahertz Center, University of Regensburg, 93040 Regensburg, Germany \\ ${ }^{2}$ Ioffe Physical-Technical Institute, Russian Academy of Sciences, 194021 St. Petersburg, Russia \\ ${ }^{3}$ Linköping University, S-58183 Linköping, Sweden \\ ${ }^{4}$ Chalmers University of Technology, S-41296 Göteborg, Sweden
}

(Received 12 August 2010; published 23 November 2010)

\begin{abstract}
We report the observation of the circular ac Hall effect where the current is solely driven by the crossed ac electric and magnetic fields of circularly polarized radiation. Illuminating an unbiased monolayer sheet of graphene with circularly polarized terahertz radiation at room temperature generates-under oblique incidence - an electric current perpendicular to the plane of incidence, whose sign is reversed by switching the radiation helicity. Alike the classical dc Hall effect, the voltage is caused by crossed $\boldsymbol{E}$ and $\boldsymbol{B}$ fields which are, however rotating with the light's frequency.
\end{abstract}

For more than a century, the Hall effect has enabled physicists to gain information on the electronic properties of matter. In Hall's original experiment [1], a clever combination of static magnetic and electric fields allowed to determine the sign and density of charge carriers, opening the door to a more thorough understanding of electronic transport in metals and semiconductors. The circular ac Hall effect (CacHE), in contrast, driven by the crossed ac $\boldsymbol{E}$ and $\boldsymbol{B}$ fields of circularly polarized light, delivers information on the underlying electron dynamics. The effect remained so far undiscovered as electromagnetic radiation incident upon low-dimensional structures causes all sorts of photocurrents stemming from both contact and band-structure specifics. With respect to the latter, the newly discovered graphene [2] is an ideal model system as symmetry prevents other helicity-driven photocurrents like the circular photogalvanic [3] or spin-galvanic effect [4] to occur. These effects require the lack of spatial inversion and are therefore forbidden in the honeycomb crystal lattice of graphene having the symmetry $D_{6 h}$ [5].

Two types of graphene were investigated: large area graphene prepared by high temperature $\mathrm{Si}$ sublimation of semi-insulating silicon carbide ( $\mathrm{SiC})$ substrates [7] and exfoliated graphene [2] deposited on oxidized silicon wafers. While both types of samples showed the effect, the micron sized exfoliated samples displayed an additional edge contribution (discussed in Ref. [6]) as the spot size of the terahertz $(\mathrm{THz})$ laser of $1 \mathrm{~mm}^{2}$ was larger than the graphene flakes. Hence, we focus on the large area $\mathrm{SiC}$ based samples having areas of $3 \times 3$ and $5 \times 5 \mathrm{~mm}^{2}$. We studied both $n$ - and $p$-type layers with carrier concentrations in the range of $(3-7) \times 10^{12} \mathrm{~cm}^{-2}$ and mobilities about $1000 \mathrm{~cm}^{2} / \mathrm{V} \mathrm{s}$ at room temperature. The experimental geometry is sketched in Fig. 1. The graphene samples were illuminated at oblique incidence, where the incidence angle $\theta_{0}$ was varied between $-40^{\circ}$ and $+40^{\circ}$.
The resulting photocurrent was measured at room temperature for wavelengths between $90 \mu \mathrm{m}$ and $280 \mu \mathrm{m}$ using either a continuous-wave (cw) $\mathrm{CH}_{3} \mathrm{OH}$ laser or a high power pulsed $\mathrm{NH}_{3}$ laser $[8,9]$. For these wavelengths the condition $\omega \tau<1$ holds, with $\omega$ the angular frequency of the light and $\tau$ the momentum relaxation time of electrons (holes) in graphene. The resulting photocurrent is measured by the voltage drop across a load resistor between pairs of contacts made at the edges of the graphene square. To prove that the signal stems from graphene and not, e.g., from the substrate, we removed the graphene layer from one of the exfoliated samples and observed that the signal disappeared. The degree of circular polarization, $P_{\text {circ }}=$ $\sin 2 \varphi$, is adjusted by a quarter-wave plate, where $\varphi$ is the angle between the initial polarization vector of the laser light and the $c$ axis of the plate.

The photocurrent for the transversal geometry, $j_{y}$, is shown in Fig. 2 as a function of $\varphi$. The principal observation made in all investigated samples is that for circularly polarized light, i.e., for $\varphi=45^{\circ}$ and $135^{\circ}$, the sign of $j_{y}$ depends on the light's helicity and the charge carriers' polarity. The overall dependence of $j_{y}$ on $\varphi$ is more complex and, at small $\theta_{0}$, well described by

$$
j_{y}=A \theta_{0} \sin 2 \varphi+B \theta_{0} \sin 4 \varphi+\xi .
$$

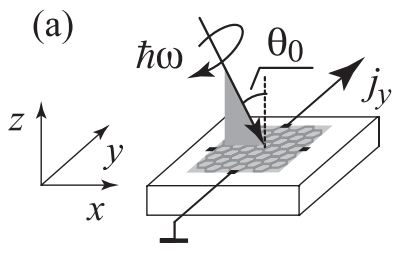

(b)

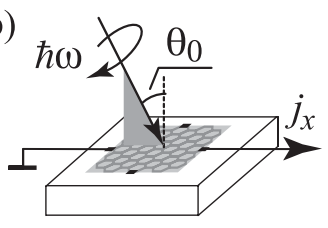

FIG. 1. Experimental configurations showing the plane of incidence of the radiation and the arrangement of contacts at the edges of graphene. Both (a) transverse and (b) longitudinal arrangements were used to measure the photocurrents. 


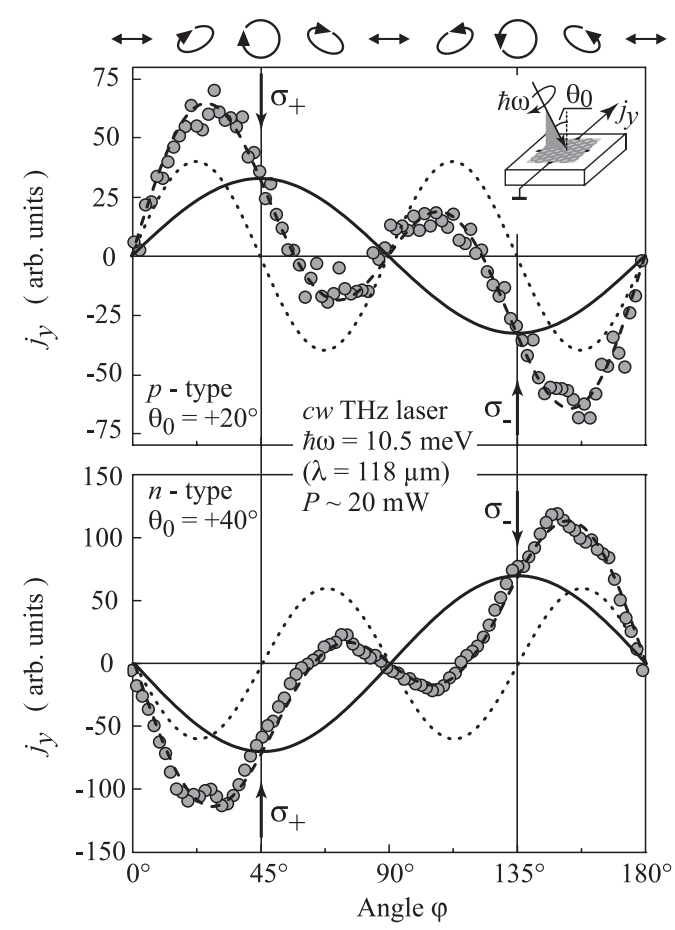

FIG. 2. Transverse photocurrent $j_{y}$ as a function of the angle $\varphi$ for $p$ - and $n$-type graphene. The ellipses on top illustrate the polarization states for various $\varphi$. Dashed lines show fits to the calculated total current $j_{A}+j_{B}$ comprising the circular contribution $j_{A}$ (CacHE, full line) and the linear contribution $j_{B}$ (dotted line). An offset $\xi, 2$ times smaller than $j_{A}$, was subtracted.

Here, $\xi$ is a polarization independent offset, ascribed to sample or intensity inhomogeneities. It does not change with the angle $\theta_{0}$ and is subtracted from the data of Fig. 2. The fit parameters $A$ and $B$ describe the strength of the circular contribution $j_{A} \propto \sin 2 \varphi$ and of the contribution $j_{B} \propto \sin 4 \varphi$ caused by linear polarization. Both contributions are shown together with the resulting fit of the data in Fig. 2. Note that for purely circularly polarized light, the linear contribution $j_{B}$ vanishes.

In the longitudinal geometry [Fig. 1(b)], only linearly polarized light gives rise to the $\varphi$ dependence of $j_{x}$ :

$$
j_{x}=B \theta_{0}(1+\cos 4 \varphi)+C \theta_{0}+\xi^{\prime} .
$$

This is shown in the inset of Fig. 3 for both $n$ - and $p$-type graphene. A sizable fraction of $j_{x}$ stems from the polarization independent contribution $j_{C}=C \theta_{0}$, whose sign does not reverse with helicity. Both currents $j_{y}$ and $j_{x}$, however, change their signs upon reversing the direction of incidence (Fig. 3).

The experimental data are well described by the theoretical model, outlined below. While the longitudinal currents can be explained along similar lines, we focus on the transverse helicity-driven current $j_{A}$. The basic physics behind the CacHE is illustrated in Fig. 4. Here, we consider the classical regime, where the photon energy is much smaller than the Fermi energy, $\hbar \omega \ll\left|E_{F}\right|$, fulfilled in the experiment as $\left|E_{F}\right|$ is $\sim 100 \mathrm{meV}$ while the photon

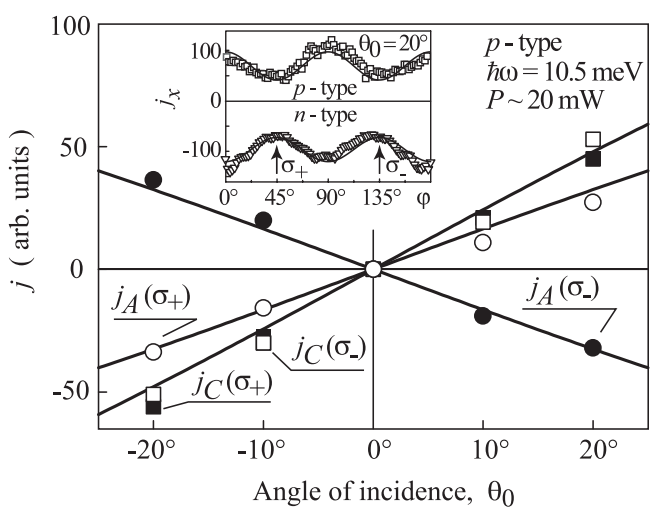

FIG. 3. Photocurrents $j_{A}$ (circles) and $j_{C}$ (squares) induced by circularly polarized light $\sigma_{ \pm}\left(\varphi=45^{\circ}\right.$ and $\left.135^{\circ}\right)$ as function of the incidence angle $\theta_{0}$. Open symbols correspond to $\sigma_{+}$, filled symbols to $\sigma_{-}$light. The solid lines are fits based on Eqs. (4) and (5). The inset shows the $\varphi$ dependence of $j_{x}$ measured in $p$-and $n$-type graphene together with fits according to Eq. (2). The constant offsets $\xi$ and $\xi^{\prime}$ have been subtracted.

energy $\hbar \omega$ is typically $\sim 10 \mathrm{meV}$. For circularly polarized radiation, the electric field rotates around the wave vector $\boldsymbol{q}$, sketched in Fig. 4(a) for $\sigma_{+}$circularly polarized light. This leads to an orbital motion of the holes (electrons) illustrated in Fig. 4. The CacHE comes into existence due to the combined action of the rotating electric and magnetic field vectors $\boldsymbol{E}$ and $\boldsymbol{B}$, respectively. At an instant of time, e.g., at $t_{1}$, the electron is accelerated by the in-plane component $\boldsymbol{E}_{\|}$of the ac electric field. At the same time, the electron with velocity $\boldsymbol{v}$ is subjected to the out-of-plane magnetic field component $\boldsymbol{B}_{z}$. Note, that the velocity $\boldsymbol{v}$ does not instantaneously follow the actual $\boldsymbol{E}_{\|}$-field direction due to retardation: There is a phase shift equal to $\arctan (\omega \tau)$ between the electric field and the electron velocity $\boldsymbol{v}$. Only for $\omega \tau \ll 1$ the directions of $\boldsymbol{v}$ and $\boldsymbol{E}_{\|}$ coincide. The effect of retardation, well known in the Drude-Lorentz theory of high frequency conductivity [10], results in an angle between the velocity $\boldsymbol{v}$ and the electric field direction $\boldsymbol{E}_{\|}$, which depends on the value of $\omega \tau$. The resulting Lorentz force $\boldsymbol{F}_{L}=e\left(\boldsymbol{v} \times \boldsymbol{B}_{z}\right)$, where $e$ is the positive (holes) or negative (electrons) carrier charge, generates a Hall current $\boldsymbol{j}$, also shown in Fig. 4. Half a period later at $t_{2}=t_{1}+T / 2$, both $\boldsymbol{v}$ and $\boldsymbol{B}_{z}$ get reversed so that the direction of $\boldsymbol{F}_{L}$ and, consequently, the current $\boldsymbol{j}$ stay the same. The oscillating magnitude and direction of $\boldsymbol{B}_{z}$ along the closed trajectory leads to a periodical modulation of the Lorentz force with nonzero average causing a nonzero time-averaged Hall current with fixed direction.

If, as shown in Fig. 4(c), the light helicity is reversed, the electric field rotates in the opposite direction and, thus, the carrier reverses its direction. Hence, the $y$-component of $\boldsymbol{F}_{L}$ at $t_{1}$ and $t_{2}$ is inverted. Consequently the polarity of the transverse, time-averaged Hall current changes. This is the circular ac Hall effect. On the other hand, we obtain the longitudinal current $j_{x}$, which does not change direction when the helicity flips. This current is also observed in 
(a) $\sigma_{+}$, right-handed radiation

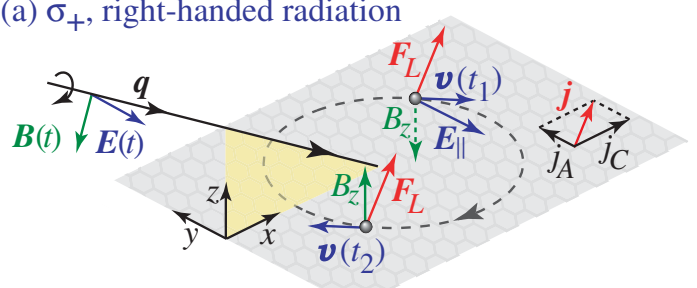

(b) $\sigma_{+}$, right-handed radiation

(c) $\sigma_{-}$, left-handed radiation
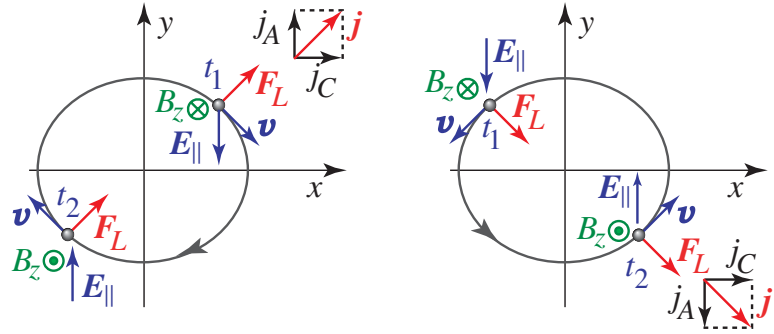

FIG. 4 (color). Schematic illustration of the circular ac Hall effect. For simplicity we assume positive carriers, i.e., holes. (a) $\boldsymbol{E}$ and $\boldsymbol{B}$ field vectors of $\sigma_{+}$polarized light with wave vector $q$ under oblique incidence in the $(x z)$ plane. The solid orbit represents the hole's elliptical trajectory caused by the ac $\boldsymbol{E}$ field. The relevant vectors are shown for two instants in time, $t_{1}$ and $t_{2}$, shifted by half a period; $\boldsymbol{v}_{1}$ and $\boldsymbol{v}_{2}$ are the hole velocities at $t_{1}$ and $t_{2}$, respectively, taking retardation into account. The direction of the Lorentz force $\boldsymbol{F}_{L}$ due to the ac $\boldsymbol{B}$ field determines the direction of the Hall current $\boldsymbol{j}$. (b) Top view of (a). (c) Same as (b) but for $\sigma_{-}$light.

our experiment, displayed in Fig. 3. Obviously, flipping the angle of incidence, $\theta_{0} \rightarrow-\theta_{0}$, results in a change of the relative sign of $\boldsymbol{E}_{\|}$and $\boldsymbol{B}_{z}$ so that both $j_{x}$ and $j_{y}$ flip directions.

While the explanation of the CacHE has been given in a pictorial way above, we resort now to a microscopic description based on the Boltzmann kinetic equation for the electron distribution function $f(\boldsymbol{p}, \boldsymbol{r}, t)$, with the freecarrier momentum $\boldsymbol{p}$, in-plane coordinate $\boldsymbol{r}$, and time $t$ :

$$
\frac{\partial f}{\partial t}+\boldsymbol{v} \frac{\partial f}{\partial \boldsymbol{r}}+e(\boldsymbol{E}+\boldsymbol{v} \times \boldsymbol{B}) \frac{\partial f}{\partial \boldsymbol{p}}=Q\{f\} .
$$

Here, $Q\{f\}$ is the collision integral described in terms of momentum relaxation times $\tau_{n}(n=1,2 \ldots)$ for corresponding angular harmonics of the distribution function $[6,11]$. The electric current density is given by the standard equation $\boldsymbol{j}=4 e \sum_{p} \boldsymbol{v} f(\boldsymbol{p})$, where a factor of 4 accounts for spin and valley degeneracies. In order to solve the kinetic Eq. (3), we expand the solution in powers of electric and magnetic fields, keeping linear and quadratic terms only. This is described in more detail in Ref. [6] closely following previous work [11]. In the calculation of $f(\boldsymbol{p})$ and $j$, we used the energy dispersion $\varepsilon_{p}= \pm v p$ of free carriers in graphene and the relation $\boldsymbol{v} \equiv \boldsymbol{v}_{p}=v \boldsymbol{p} /|\boldsymbol{p}|$ between the velocity and the quasimomentum $(v \approx$ $c / 300$, with $c$ being the speed of light). Contributions to the photocurrent appear not only from a combined action of the electric and magnetic fields of the light wave, illustrated in Fig. 4, but also due to the spatial gradient of the electric field [11]. As final result we obtain for the helicity-driven current

$$
j_{A}=A \theta_{0} \sin 2 \varphi=q \theta_{0} \chi P_{\text {circ }}\left(1+\frac{\tau_{2}}{\tau_{1}}\right) \frac{1-r}{1+\omega^{2} \tau_{2}^{2}},
$$

flowing in $y$-direction, and the $\varphi$-independent current

$$
j_{C}=C \theta_{0}=\frac{q \theta_{0} \chi}{\omega \tau_{1}}\left[2(1+r)+(1-r) \frac{1-\omega^{2} \tau_{1} \tau_{2}}{1+\omega^{2} \tau_{2}^{2}}\right] \text {, }
$$

flowing along $x$ (for light propagating in the ( $x z)$ plane). Here $q=\omega / c, q \sin \theta_{0} \approx q \theta_{0}, r=d \ln \tau_{1} / d \ln \varepsilon$ and $\chi=$ $e^{3} \tau_{1}\left(v \tau_{1} E\right)^{2} /\left[2 \pi \hbar^{2}\left(1+\omega^{2} \tau_{1}^{2}\right)\right]$.

The results of the calculation are shown in Figs. 3 and 5. The used fitting parameters only depend on details of the underlying scattering mechanism discussed below. Equation (3) provides in addition to $j_{A}$ and $j_{C}$ also currents $j_{B, x} \propto q \theta_{0}(1+\cos 4 \varphi)$ and $j_{B, y} \propto q \theta_{0} \sin 4 \varphi$, for details see [6]. However, for circularly polarized light $\left(\varphi=45^{\circ}\right.$ or $\left.135^{\circ}\right)$, the degree of linear polarization is zero and the corresponding currents vanish leaving the undisturbed CacHE contribution.

As seen in experiment the polarity of the photocurrents is opposite for $n$ - and $p$-type graphene samples. This is expected from theory since (i) the ac Hall current $j_{y}$ as well as the longitudinal current $j_{x}$ are proportional to $e^{3}$ and (ii) the conduction- and valence-band, in the massless Dirac model, are symmetric with respect to the Dirac point.

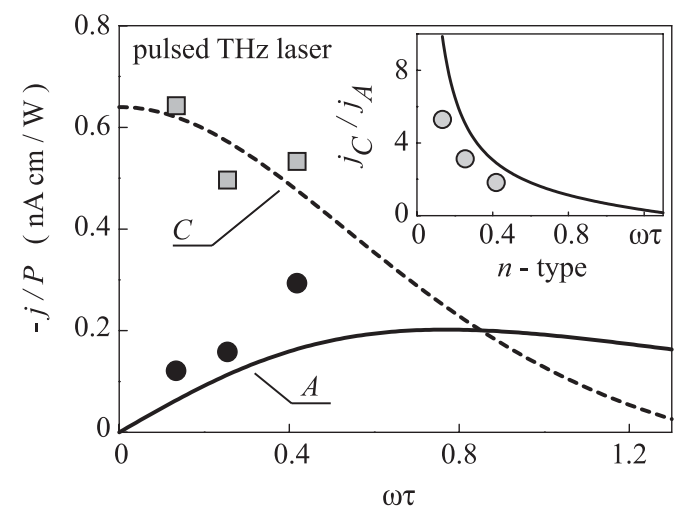

FIG. 5. Frequency dependence of $A=j_{A} / \theta_{0}$ (dots) and $C=$ $j_{C} / \theta_{0}$ (squares) as function of $\omega \tau$ for circularly polarized light. Data are shown for wavelengths 90, 148 and $280 \mu \mathrm{m}$ with the power ranging from 10 to $30 \mathrm{~kW}$. The photocurrent $j_{C}$ is obtained from the current in $x$ direction, which for $\sigma_{+}$, $\sigma_{-}$-light reads $j_{x}=C \theta_{0}$. The calculated frequency dependence of $j_{A}$ [Eq. (4), solid line] and $j_{C}$ [Eq. (5), dashed line] describe the experiment quantitatively well. The inset shows $j_{A} / j_{C}$ both for experiment and theory. This plot, independent of the absolute values, shows that the helicity-driven current $j_{A}$ vanishes for $\omega \tau \ll 1$. 
In contrast, in typical semiconductors conduction-band and valence-band states have different symmetry properties and the relation between values and polarities of the ac Hall photocurrents is more involved.

Equations (4) and (5) suggest a nonmonotonous frequency dependence of the photocurrents. In Fig. 5 the calculated frequency dependence of both $A=j_{A} / \theta_{0}$ and $C=j_{C} / \theta_{0}$ are compared quantitatively to experimental data. For the momentum scattering time we used the relation $\tau_{1}=2 \tau_{2} \propto \varepsilon_{p}^{-1}$, valid for short range scattering [12] and relevant for our low mobility samples $\left(\tau_{1} \approx 2 \times\right.$ $10^{-14} \mathrm{~s}$ ). Apart from the above assumption of short range scattering, no fit parameter was used. Figure 5 shows that the theory describes the frequency dependence and the absolute value of the photocurrent very well. Both $j_{A}$ and $j_{C}$ contribute to the photocurrent for circularly polarized light. It is remarkable that the helicity-driven current $j_{A}$ and the polarization independent photocurrent $j_{C}$ show completely different frequency dependencies. While $j_{C}$ does not change much for $\omega \tau \ll 1, j_{A}$ increases with growing $\omega \tau$ at low frequencies. For large $\omega \tau$ well above unity both photocurrents decrease with increasing $\omega$. This property agrees with the model addressed above. The CacHE, i.e. $j_{A}$, disappears for $\omega \rightarrow 0$, since no circular polarization exists for static fields and the required retardation vanishes. With increasing $\omega$ the retardation becomes important and the current increases $\propto \omega \tau$. For $\omega \tau \simeq 1$ the current gets maximal and decreases rapidly at higher $\omega, j_{y} \propto 1 / \omega^{4}$. In contrast, the longitudinal current $j_{C}$ does not depend on the frequency at $\omega \tau \ll 1$ and displays its maximum at $\omega \rightarrow 0$. The effect of retardation is just opposite to that on $j_{A}$ : Increasing $\omega$ reduces the $y$ component of the velocity (Fig. 4) and hence the $x$ component of the Lorentz force. As a consequence, $j_{C}$ drops with increasing $\omega$, see Fig. 5. The ratio of $j_{C}$ and $j_{A}$ is plotted in the inset of Fig. 5 showing that the role of the circular effect substantially increases with $\omega \tau$. The excellent agreement of theory and experiment shows that the model covers the essential physics of the circular ac Hall effect.

The photocurrents $j_{C}$ and $j_{A}$ are both proportional to the wavevector $q$ and may, therefore, also be classified as photon drag effect. In fact, the polarization independent longitudinal current $j_{C}$ is the well-known linear photon drag effect, which was first treated by Barlow [13] in 1954, observed in bulk cubic semiconductors $[14,15]$ and recently discussed for graphene $[6,16]$. The effect, described here, can be considered as the classical limit $\left(\hbar \omega \ll E_{F}\right.$ and $\omega \tau \lesssim 1$ ) of the circular photon drag effect. As it can be described in terms of the Lorentz force [see Eq. (3)], we call it the ac Hall effect. The circular photon drag effect, which takes over at higher frequencies, i.e., for $\omega \tau \gg 1$, was discussed phenomenologically $[17,18]$ and observed in GaAs quantum wells in the midinfrared range [19]. In this pure quantum mechanical limit the picture above is inapplicable and involves asymmetric optical transitions and relaxation in a spin polarized nonequilibrium electron gas. The drag effect in metallic photonic crystals, generating a transverse current due to microscopic voids, was reported recently [20].

The appearance of a helicity-driven Hall current is a specific feature of two-dimensional, even centrosymmetric, structures like graphene. CacHE is a general phenomenon and should exist in any low-dimensional system. It is, however, more readily observable in a monoatomic layer like graphene, as in multilayered low-dimensional systems, e.g., quantum wells, the CacHE is masked by the circular photogalvanic effect [3].

We thank J. Fabian, V. V. Bel'kov, J. Kamann, and V. Lechner for fruitful discussions and support. Support from DFG (SPP 1459 and GRK 1570), Linkage Grant of IB of BMBF at DLR, RFBR, Russian Ministry of Education and Sciences, President grant for young scientists and "Dynasty" Foundation ICFPM is acknowledged.

[1] E. H. Hall, Am. J. Math. 2, 287 (1879).

[2] K. S. Novoselov et al., Science 306, 666 (2004).

[3] E. L. Ivchenko and S.D. Ganichev, in Spin Physics in Semiconductors, edited by M.I. Dyakonov (Springer, New York, 2008).

[4] S. D. Ganichev et al., Nature (London) 417, 153 (2002).

[5] A more detailed symmetry analysis taking into account the possible symmetry breaking by the substrate of graphene samples can be found in Ref. [6].

[6] J. Karch et al., arXiv:1002.1047v1.

[7] A. Tzalenchuk et al., Nature Nanotech. 5, 186 (2010).

[8] S. D. Ganichev and W. Prettl, Intense Terahertz Excitation of Semiconductors (Oxford Univ. Press, Oxford, 2006).

[9] S. D. Ganichev, S. A. Emel'yanov, and I. D. Yaroshetskii, Pis'ma Zh. Eksp. Teor. Fiz. 35, 297 (1982) [JETP Lett. 35, 368 (1982)].

[10] N. W. Ashcroft and N. D. Mermin, Solid State Physics (Brooks/Cole Thomson Learning, Singapore, 2009).

[11] V. I. Perel' and Ya. M. Pinskii, Sov. Phys. Solid State 15, 688 (1973).

[12] S. Das Sarma et al., arxiv:1003.4731v1 [Rev. Mod. Phys. (to be published)].

[13] H. M. Barlow, Nature (London) 173, 41 (1954).

[14] A. M. Danishevskii et al., JETP 31, 292 (1970).

[15] A. F. Gibson et al., Appl. Phys. Lett. 17, 75 (1970).

[16] M. V. Entin, L. I. Magarill, and D. L. Shepelyansky, Phys. Rev. B 81, 165441 (2010).

[17] E. L. Ivchenko and G. E. Pikus, in Problems of Modern Physics, edited by V. M. Tuchkevich and V. Ya. Frenkel (Nauka, Moscow, 1980); Semiconductor Physics, edited by V. M. Tuchkevich and V. Ya. Frenkel (Cons. Bureau, New York, 1986).

[18] V. I. Belinicher, Sov. Phys. Solid State 23, 2012 (1981).

[19] V. A. Shalygin et al., JETP Lett. 84, 570 (2006).

[20] T. Hatano et al., Phys. Rev. Lett. 103, 103906 (2009). 